Full Research Paper

\title{
Development of a Fully Automated, GPS Based Monitoring System for Disaster Prevention and Emergency Preparedness: PPMS $^{+\mathrm{RT}}$
}

\author{
Jason Bond *, Don Kim , Adam Chrzanowski and Anna Szostak-Chrzanowski \\ Department of Geodesy and Geomatics Engineering, University of New Brunswick, 15 Dineen Drive, \\ Fredericton, New Brunswick, Canada; E-mail: jason.bond@unb.ca, kim@unb.ca, adamc@unb.ca, \\ amc@unb.ca \\ * Author to whom correspondence should be addressed.
}

Received: 29 May 2007 / Accepted: 26 June 2007 / Published: 28 June 2007

\begin{abstract}
The increasing number of structural collapses, slope failures and other natural disasters has lead to a demand for new sensors, sensor integration techniques and data processing strategies for deformation monitoring systems. In order to meet extraordinary accuracy requirements for displacement detection in recent deformation monitoring projects, research has been devoted to integrating Global Positioning System (GPS) as a monitoring sensor. Although GPS has been used for monitoring purposes worldwide, certain environments pose challenges where conventional processing techniques cannot provide the required accuracy with sufficient update frequency. Described is the development of a fully automated, continuous, real-time monitoring system that employs GPS sensors and pseudolite technology to meet these requirements in such environments. Ethernet and/or serial port communication techniques are used to transfer data between GPS receivers at target points and a central processing computer. The data can be processed locally or remotely based upon client needs. A test was conducted that illustrated a $10 \mathrm{~mm}$ displacement was remotely detected at a target point using the designed system. This information could then be used to signal an alarm if conditions are deemed to be unsafe.
\end{abstract}

Keywords: GPS, Deformation Monitoring, Kalman Filter, Wireless Network, Pseudolites 


\section{Introduction}

Disasters such as landslides, avalanches and structural failures do not occur without warning. Through strategic monitoring of the deformable object, abnormal behaviour can be detected and used to warn of imminent failure. The increasing number of structural collapses, slope failures and other natural disasters has lead to a demand for new sensors, sensor integration techniques and data processing strategies for deformation monitoring systems.

The Canadian Centre for Geodetic Engineering (CCGE) at the University of New Brunswick (UNB) is involved in the development of new methods and techniques for integrated monitoring, integrated analysis, and prediction (deterministic modeling) of structural and ground deformations. Recent, large scale, deformation monitoring projects in open pit mines have placed extraordinary demands for displacement detection accuracy.

In large open pit mines and in other steep embankment areas, the stability of steep walls is a major safety issue. In general, there is slow movement of the rock formation followed by acceleration before a failure occurs. With continuous monitoring of creep and acceleration of the rock movement, warning can be given to evacuate equipment and personnel in advance of a failure. In order to perform such tasks, monitoring accuracies of up to $\pm 5 \mathrm{~mm}$ with $95 \%$ confidence have been requested.

These requirements can be extremely difficult to meet since the achievable precision of geodetic technologies can be severely limited by the characteristics of an open pit. It is not uncommon for the degradation in precision of geodetic technologies to be so large that the minimum detectable displacement exceeds the mine's requirements. For this reason, large open pit mines may be considered one of the most challenging deformation monitoring scenarios.

For example, in large scale projects where the pit diameter exceeds $1 \mathrm{~km}$, refraction and pointing errors limit the effectiveness of direction measurements made by total station instruments and laser scanners [1]. The steep pit walls limit the effectiveness of space borne interferometric, synthetic aperture radar [2]. The steep pit walls also limit the effectiveness of satellite positioning technologies by diluting the geometric strength of solutions and reducing the frequency at which updates can be provided. Additionally, large height differences between master and rover stations can lead to significant height biases in baseline solutions [1].

This research focuses on providing fully automated, continuous, high precision position updates using one of the above mentioned geodetic technologies: the Global Positioning System (GPS). Although GPS has been used for monitoring purposes worldwide, certain environments pose challenges where conventional processing techniques cannot provide the required accuracy with sufficient update frequency. Discussed are the challenges encountered in using GPS in harsh environments and the innovations that have been introduced in order to successfully integrate this technology for deformation monitoring in such scenarios. 


\section{Using GPS for Deformation Monitoring in Harsh Environments}

GPS (and in general, Global Navigation Satellite Systems (GNSS)) offers potential advantages over other geodetic technologies that allows for continuous and high accuracy displacement detection. These include:

a) Line of sight is not required between stations;

b) Updates can be provided at frequencies of $1 \mathrm{~Hz}$ and higher;

c) 3 dimensional position information is provided; and

d) Millimetre level position information is possible for baselines potentially up to $10 \mathrm{~km}$ in length.

The major disadvantage of using GPS is the cost of a geodetic grade receiver and antenna. This will ultimately limit the number of units that can be afforded and dictate the spatial resolution of targets that can be monitored using this technology. In most cases it will be more economical to integrate GPS to monitor the stability of other sensors that can provide higher spatial resolution at a cheaper cost in localized areas (e.g., total stations, laser scanners).

To determine if the above advantages of GPS could be realized in a harsh environment, GPS data were collected at 5 stations in a large open pit mine as illustrated in Figure 1. Stations 424 and 987 were reference stations, while stations RTS1, RTS2 and RTS3 were monitored stations within the pit. Continuous data for 5 days or more were collected.

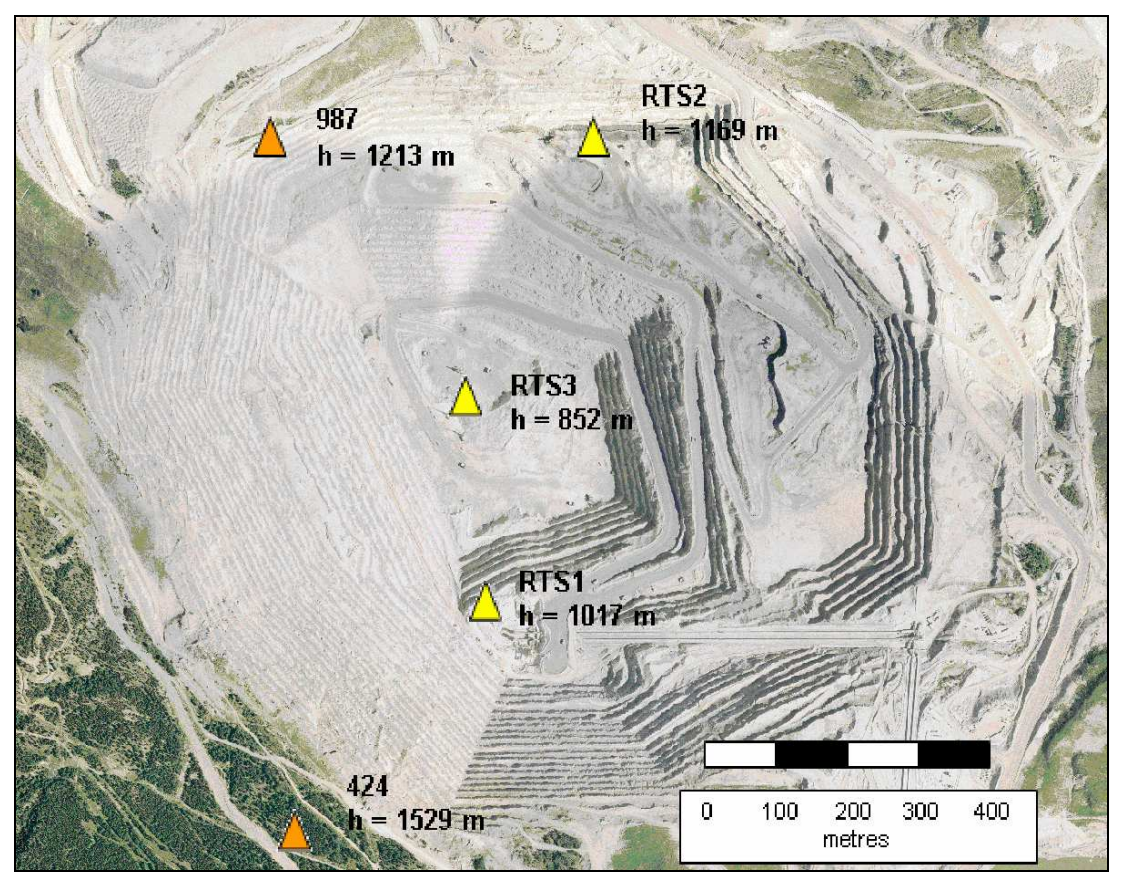

Figure 1. Large Open Pit Mine Test (with station heights shown).

Initial analyses of the GPS data were conducted using traditional batch processing methods in commercial and scientific software [1,3]. To illustrate, 3 hour, commercial software results are presented in Figure 2 showing the 'up' (height) component solutions (generally the poorest precision) for baseline 424 to RTS2. RTS2 represents the best case scenario of the monitored stations in terms of 
satellite visibility and height differences with reference stations. The results have been obtained using conventional double-differenced, L1 carrier phase processing methods. The height difference between master and rover stations is 361 meters. It can be seen that a peak-to-peak spread of over $4 \mathrm{~cm}$ exists. Since there is a large height difference, the unmodelled portion of the atmosphere between the stations causes biases in the height component as meteorological conditions vary from day to day. The RMS error for this sample is $\pm 9 \mathrm{~mm}$. There are also 4 sessions for which a fixed position solution could not be obtained due to poor data quality. Results for stations RTS1 and RTS3 show larger biases in the height component and more solution outages.

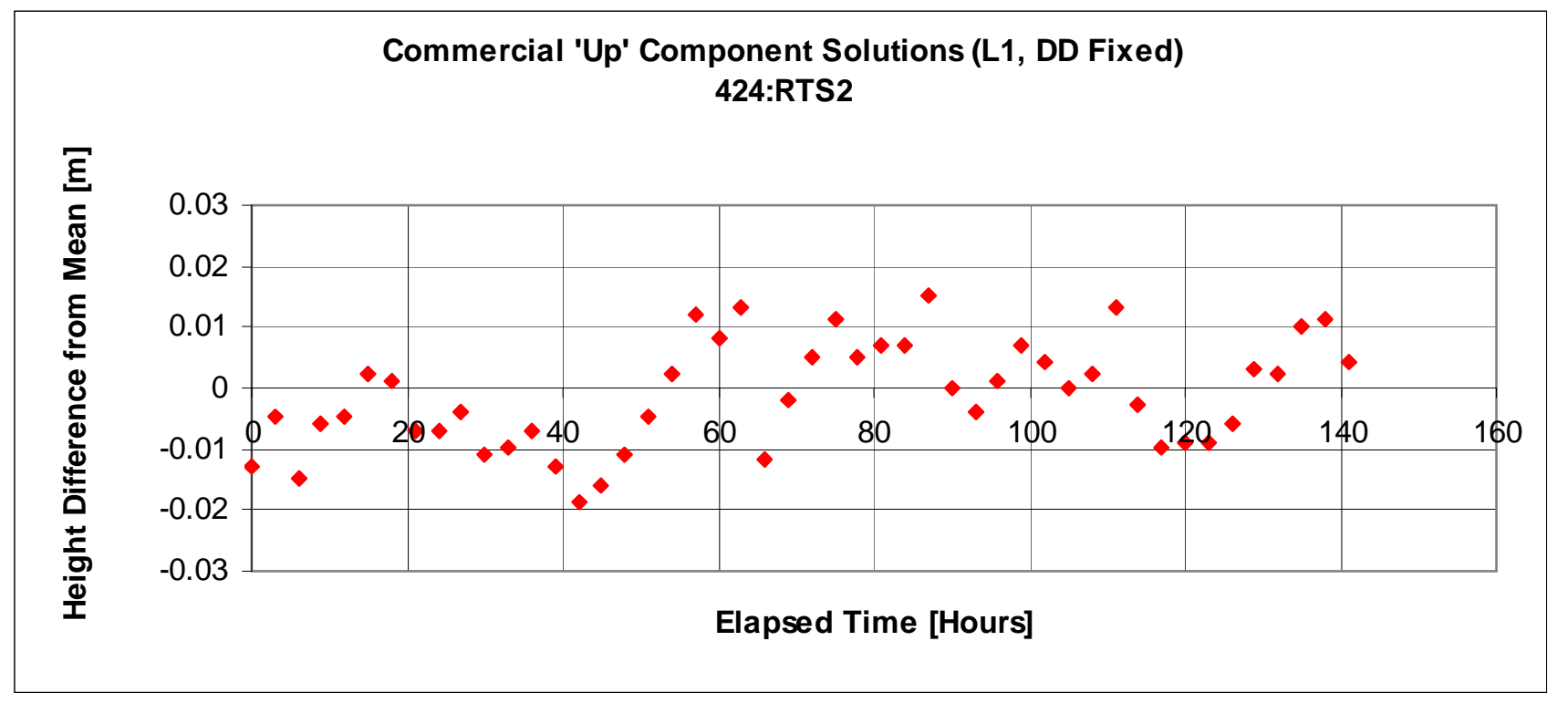

Figure 2. Commercial, 3 Hour, DD Fixed, 'Up' Component Solutions.

Further investigation was conducted to determine if longer session lengths would improve the quality of the position of estimates [1]. Other commercial and scientific (UNB's Differential Positioning Package- DIPOP, University of Bern's Bernese) software were also tested. Although the precision of the results improved with longer session lengths, the delay between position updates (sometimes up to 24 hours) would not satisfy project requirements.

From the above results, it is apparent that in order to use GPS to provide sub-centimetre displacement detection with $95 \%$ confidence, there are some major challenges that need to be addressed. Additional obstacles arise when attempting to provide fully automated updates for continuous monitoring of target points. The major challenges involved in using GPS for deformation monitoring in harsh deformation monitoring environments are:

a) Mitigating residual troposheric delay biases: Residual tropospheric delay is the portion of tropospheric delay that remains after modeling. Due to large height differences between master and rover stations, residual tropospheric delay biases can contaminate the vertical component of GPS baseline solutions. Beutler et al. [4] have illustrated that neglecting the differential troposphere causes a 3-5 mm relative height error for every millimetre of difference in zenith delay between stations. Estimating residual tropospheric delay bias terms is the usual approach 
to improving accuracy in such situations. The strength of the bias estimates, however, depends upon low elevation satellites which are generally not visible [5]. In order to meet subcentimetre accuracy requirements in large open pit mines using GPS, a new methodology is required to mitigate this bias.

b) Providing continuous updates with limited satellite visibility: The steep pit walls of open pit mines obstruct satellite visibility. This limits the reliability of the solutions as well as the frequency at which updates can be provided. In order to meet sub-centimetre accuracy requirements with sufficient update frequency, new technologies must be integrated with GPS.

c) Connecting to stable reference points: As precise as the GPS software may be, the overall accuracy of the solution depends upon the validity of the assumption that each reference point is stable. Tremendous care must be taken in choosing suitable reference station locations. Additional sources of information regarding the properties of the rock mass must be utilized to make informed decisions.

d) Developing a fully automated GPS processor: A fully automated GPS processor is required to provide continuous updates in real-time. Ideally, the results from the processor can be used to provide 'on-time' warnings of impending danger. The processor must be designed to be robust so that false alarms do not occur. Additionally, the precision of the solutions must satisfy subcentimetre displacement detection requirements with 95\% confidence. Communication links must also be built into the software to allow for data transfer between GPS receivers located on site at target points and a central processing computer.

\section{Overcoming GPS Deformation Monitoring Challenges}

In order to capitalize on the benefits of GPS, the aforementioned challenges need to be addressed. Research has been devoted to answering each of these needs [6-8]. Discussed are the innovations introduced to overcome these obstacles.

\subsection{Mitigating Residual Tropospheric Delay}

A common technique of eliminating biases is through differencing of observations. For example, between-receivers differencing (SD: single difference) of GPS observations to the same satellite eliminates satellite clock biases and between-satellite differencing of GPS observations to the same receiver eliminates receiver clock biases. Further differencing between-receivers SDs and betweensatellite SDs results in a double-differenced (DD) observable free of both receiver clock and satellite clock biases.

The devised methodology is based upon time differencing of successive DD observations and gives the triple-differenced (TD) observable [9]. The appeal of the TD observable to most users is its absence of the integer ambiguity term which cancels out through the time difference if a cycle slip has not occurred. Although the TD observable is not new, obtaining high precision results in environments 
where the DD observable suffers from residual tropospheric delay biases presents another beneficial use.

After removing the error terms for receiver clocks and satellite clocks (which should be eliminated through DD processing when observation simultaneity criteria are met), the TD, carrier-phase observable between times $t_{1}$ and $t_{2}$ can be written as:

$$
\begin{aligned}
\delta{\underset{A B}{\Delta}}_{\nabla}^{i j} \varphi\left(t_{12}\right) & =\left[\underset{A B}{\Delta} \stackrel{i j}{\nabla}\left(\varphi+N+M-\frac{f}{c} I+\frac{f}{c} T+e_{\varphi}\right)+\varepsilon_{\text {trop }}\right]_{t_{2}} \\
- & {\left[\underset{A B}{\Delta} \stackrel{i j}{\nabla}\left(\varphi+N+M-\frac{f}{c} I+\frac{f}{c} T+e_{\varphi}\right)+\varepsilon_{\text {trop }}\right]_{t_{1}} }
\end{aligned}
$$

where:

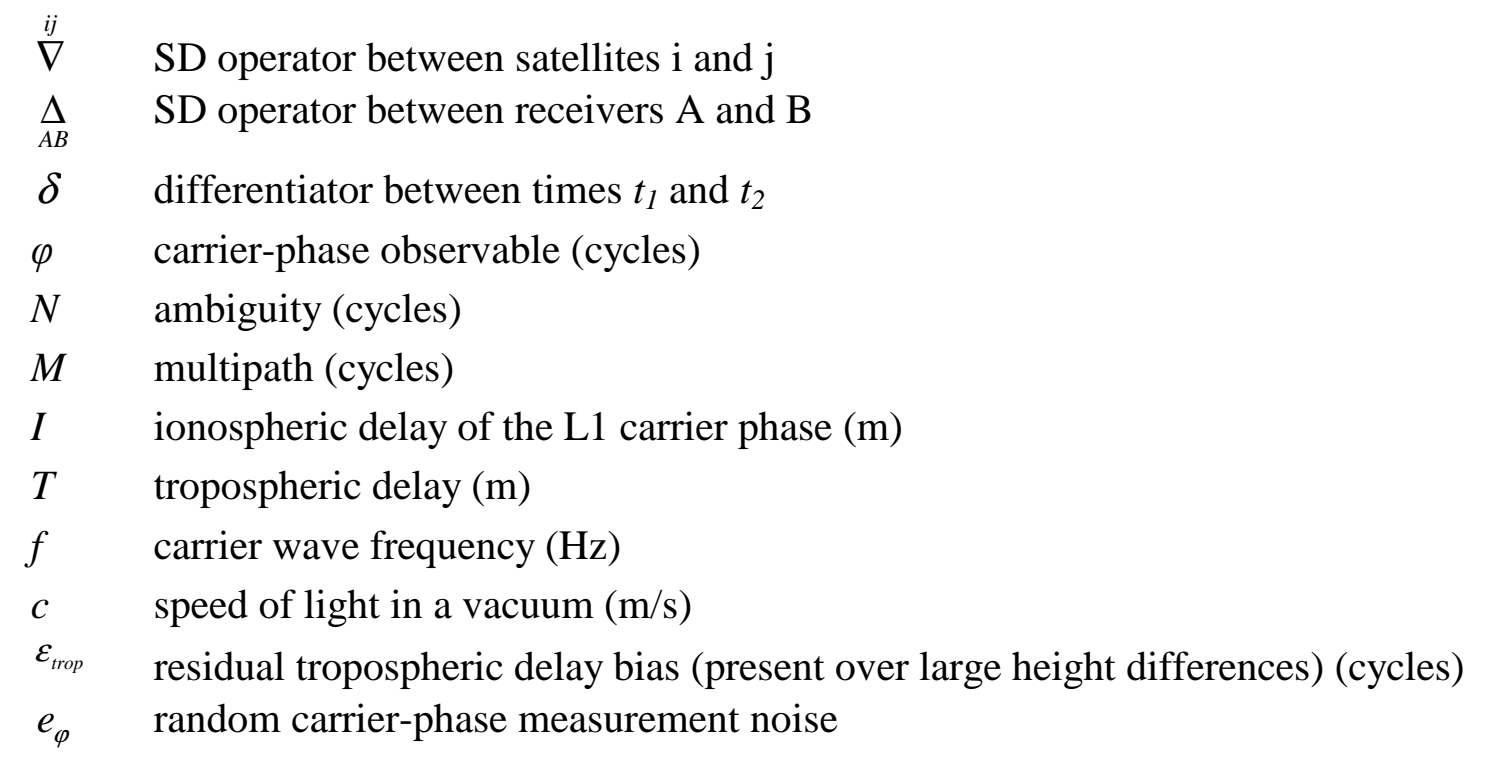

For observation intervals less than a few seconds, the correlation in atmospheric parameters between times $t_{1}$ and $t_{2}$ is large and therefore biases originating from them are significantly reduced. Since the ambiguity term $N$ cancels, Equation (1) can be rewritten as:

$$
\delta \underset{A B}{\Delta} \stackrel{i j}{\nabla} \varphi\left(t_{12}\right)=\left[\Delta \Delta_{A B}^{i j}(\varphi+M)\right]_{t_{2}}-\left[\Delta \Delta_{A B}^{i j}(\varphi+M)\right]_{t_{1}}+e_{\delta \Delta \nabla \varphi}
$$

where:

$$
e_{\delta \Delta \nabla \varphi} \text { random noise error of a TD carrier-phase observation }
$$

The TD observable may be considered as an integrated (radial) velocity measurement which is used to determine position. The measurement form is [10]:

$$
\begin{aligned}
(\text { measurement })_{k}= & \int_{t_{k-1}}^{t_{k}}(\text { velocity }) d t+\text { noise } \\
& =(\text { position })_{k}-(\text { position })_{k-1}+\text { noise }
\end{aligned}
$$


In mathematical form, the measurement equation can be written as:

$$
z_{k}=H_{k} x_{k}+J_{k} x_{k-1}+v_{k}
$$

where $H_{k}\left(=-J_{k}\right)$ is a directional cosine matrix.

This form does not fit the general format for the usual Kalman filter equations due to the $x_{k-1}$ term. The general Kalman filter equations must be modified to accommodate this term as well as any correlation in process and measurement noise. The modified version is known as the Delayed-State Kalman filter. The interested reader can find the Delayed-State equations in [6, 10].

Recently, the Precise Position Monitoring System software has been developed that employs a Delayed-State Kalman filter to process triple-differenced carrier phase observations [6]. Initial test results have been promising, showing that $\mathrm{mm}$ level precisions are attainable in horizontal and vertical position components without having to solve for the ambiguity term. The expense of using this technique is slow filter convergence time due to the nature of the TD observable (it reflects the change in satellite geometry from one epoch to the next). This is generally not a concern for long term deformation monitoring applications.

Figure 3 presents the east, north and up components of the 424:RTS2 baseline processed using PPMS. There is a height change of a few $\mathrm{mm}$ that is detected after hour 50, which is not so easily identified in Figure 2. The peak-to-peak spread of the up component in Figure 3 is in the order of 1 $\mathrm{cm}$ after the change in height, as opposed to $4 \mathrm{~cm}$ in Figure 2. This can be attributed to the effects of residual tropospheric delay being reduced by the TD approach. The improved precision combined with more continuous updates allows for a clearer picture of the deformation behavior of the target point. A comparison of DD batch processing with the TD Delayed-State Kalman filter approach is presented in Table 1.

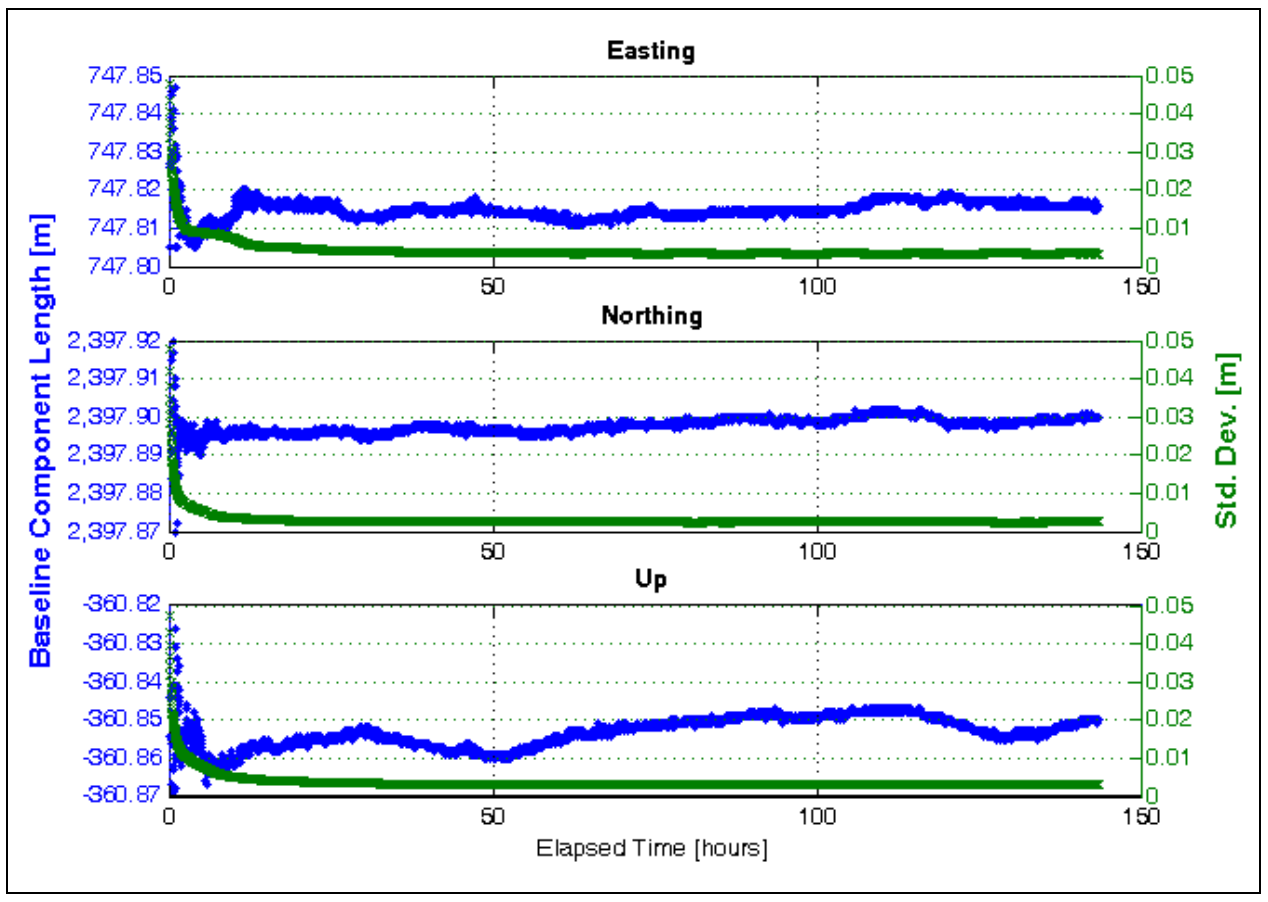

Figure 3. Continuous, TD, east, north, up solutions using PPMS. 


\begin{tabular}{lll}
\hline Processing Strategy & \multicolumn{1}{c}{ Advantages } & \multicolumn{1}{c}{ Disadvantages } \\
\hline DD Batch Processing & - High precision results & - Results are not continuous \\
& - Lower level of differencing & - Height biases caused by residual \\
& results in less observation & tropospheric delay over large \\
noise & & $\begin{array}{l}\text { height differences when low } \\
\text { elevation satellites are not } \\
\text { visible }\end{array}$ \\
& & - Slow convergence time \\
TD Kalman Filter & - High precision results & - Increased observation noise \\
& - Results provided at sample & caused by additional level of \\
& rate & differencing \\
& - Significantly reduces & \\
& atmospheric biases (residual & \\
& tropospheric delay) over high & \\
& sample rates & \\
& - Less chance of false alarms \\
& caused by cycle slips & \\
&
\end{tabular}

Table 1. Comparison of DD Batch Processing with TD Kalman Filter Processing Methods.

\subsection{Improving Continuity in Solution Updates}

Analysis of the GPS data obtained from the previously described large open pit mine indicated that there were several periods during the day for which fewer than 4 satellites were visible. This leads to degradation in the precision of the solutions as well as a reduction in the frequency of updates. Consequently, the potential of augmenting GPS with pseudolites (PLs) has been investigated to improve the frequency and precision of solutions [7]. The devised approach incorporates the PL observations into the TD Delayed-State Kalman Filter.

Being a ground-based signal transmitter, PL error sources must be handled differently than GPS signal error sources. PPMS was modified to address nuances in PL data processing which include cycle slip detection, PL location determination and PL observation modelling. As before, after removing the error terms for receiver clocks and satellite clocks (which are eliminated through double-differencing over short baselines), the TD carrier phase observable for PLs can be written in the form of Equation

$\delta \underset{A B}{\Delta} \stackrel{i P L}{\nabla} \varphi\left(t_{12}\right)=\left[\underset{A B}{\stackrel{i P L}{\nabla}}\left(\varphi+N+M-\frac{f}{c} I+\frac{f}{c} T+e_{\varphi}\right)+\varepsilon_{\text {trop }}\right]_{t_{2}}-\left[\stackrel{i P L}{\nabla}\left(\varphi+N+M-\frac{f}{c} I+\frac{f}{c} T+e_{\varphi}\right)+\varepsilon_{\text {trop }}\right]_{t_{1}}^{*}$

\footnotetext{
* There is not an ionospheric delay term for the PL observation since its signal does not travel through the ionosphere, but the reference satellite observation will include this term.
} 
The attractiveness of the TD PL observable is that it is a time difference of DD observations and consequently biases common to both observations will cancel. This strategy allows for smooth integration of PL data into the existing software infrastructure due to the following benefits:

a) the user no longer needs to solve for the ambiguity term, which allows the system to be less susceptible to biases caused by cycle slips;

b) for observation intervals less than a few seconds, the correlation between PL tropospheric delay parameters at times $t_{1}$ and $t_{2}$ will be large and biases originating from them will be significantly reduced;

c) for observation intervals less than a few seconds, the correlation between low frequency multipath terms at times $t_{1}$ and $t_{2}$ will be large and biases originating from them will be significantly reduced. The high frequency component still remains;

d) since the PL is stationary between times $t_{1}$ to $t_{2}$ and it is assumed that the receivers are stationary, the geometric distance terms involving the PL cancel so that the computed, geometric, TD is solely dependent upon the change in the geometry of the reference satellite. Consequently, the accuracy to which the PL coordinates must be known is very forgiving. Approximate coordinates are necessary simply for computing elevation angles to PLs if an elevation cut off constraint is imposed.

Figure 4 illustrates the results of a baseline that was observed in a harsh environment (having a 35 degree elevation cut-off) while using a PL. A slow, $10 \mathrm{~mm}$ displacement occurs in the horizontal plane beginning at hour 14 and ending at hour 18 as indicated by the green and red vertical lines respectively. It takes approximately 10 hours for the solution to converge. It can be seen that the combined GPS+PL system allows for more continuous solution updates and quicker displacement detection than standalone GPS.
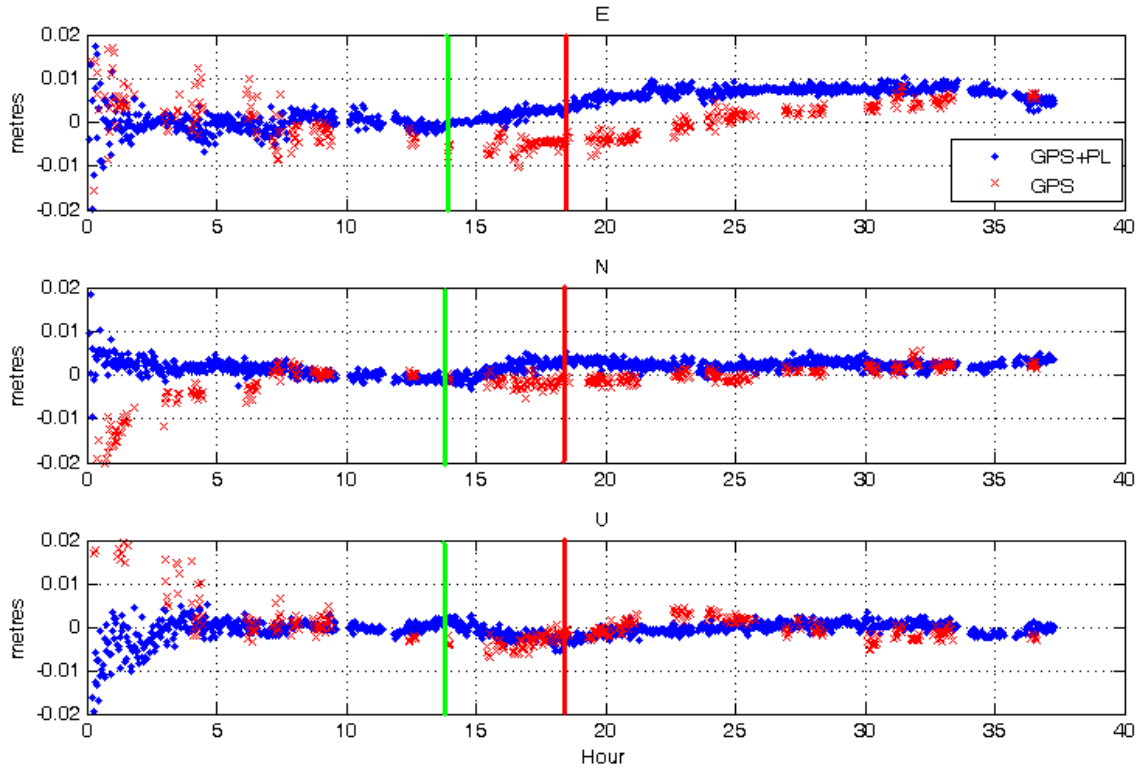

Figure 4. Detected Displacements with and without PL observations. 


\subsection{Predicting Station Behaviour using Deterministic Modelling}

Analysis of PPMS results of the GPS baselines observed in the open pit raised two important questions regarding station stability: a) is it reasonable to expect upward movements of the monitored stations located at discrete points along the pit walls as was detected by the software and b) are the reference stations stable? To answer these questions, research has been devoted to predicting deformation behaviour of the open pit and its surroundings using deterministic modelling [8]. Deterministic modelling takes into account the material properties and geometry of the deformable object, loading conditions and the physical laws governing the behaviour of the deformable object to predict displacement patterns. In most cases the closed form solution is difficult to obtain and therefore numerical methods are used.

Figure 5 illustrates the predicted displacement field in an open pit mine that occurs as a result of excavating a layer of rock along the left pit wall and pit floor. The results were obtained using the finite element method. This displacement pattern is very useful for addressing the two questions raised. First, it can be used in delineating the zone of significant deformation so that locations for stable reference points can be recommended. Additionally, this information provides an indication of the expected magnitude and direction of the displacement vectors. These results validate that it is possible that a target point located along the pit walls can experience upward movement as was captured in Figure 3.

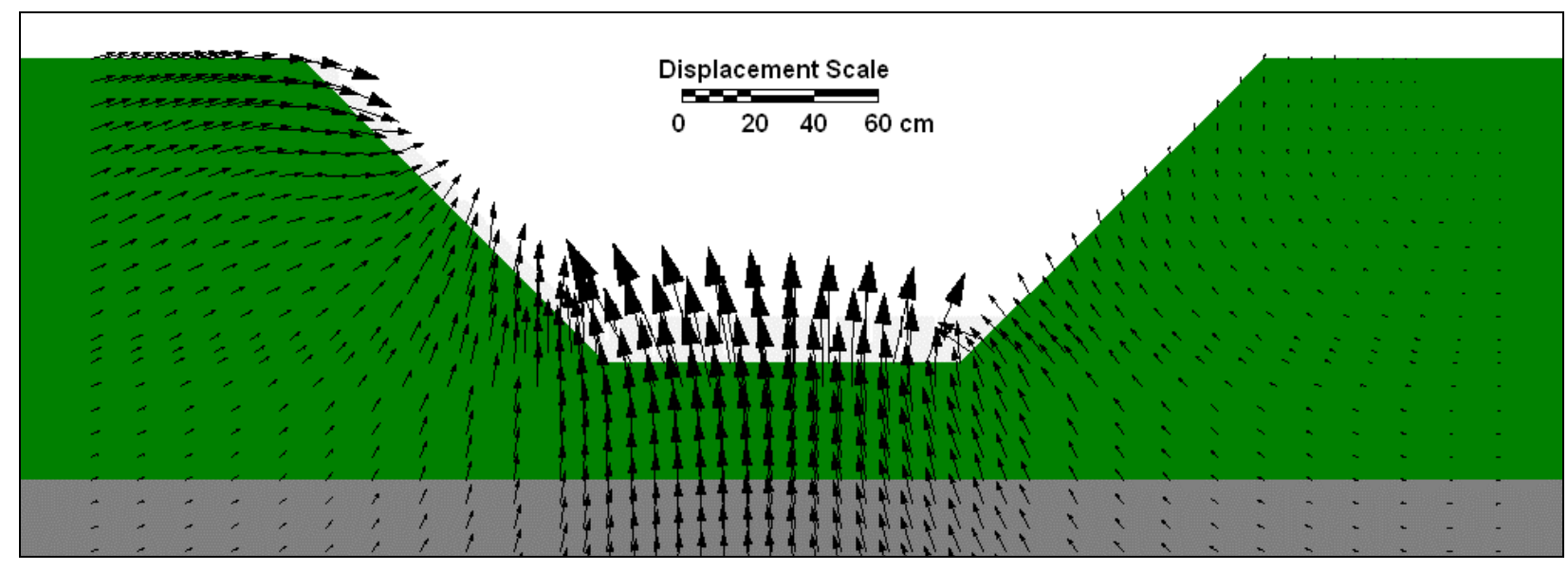

Figure 5. Predicted Displacement Pattern as a Result of Excavating the Left Pit Wall and Pit Floor.

\subsection{Developing a Fully Automated, GPS Monitoring System}

To take full advantage of the capabilities of PPMS for deformation monitoring, a fully automated version has been created: the Precise Position Monitoring System plus Real-Time $\left(\mathrm{PPMS}^{+\mathrm{RT}}\right)$. Discussed are some of the practical issues involved in the development of the real-time version of the software. A displacement detection test is presented illustrating the potential of $\mathrm{PPMS}^{+\mathrm{RT}}$ in a deformation monitoring scenario. 


\subsubsection{Monitoring System Infrastructure}

PPMS $^{+\mathrm{RT}}$ currently accepts two methods of data communication between GPS sensors and the central processing computer: (1) via Ethernet connection (either a Local Area Network (LAN) or Wireless LAN (WLAN)) and (2) via serial port (either directly or through radio modem). A monitoring system may be designed to include all Ethernet connections, all serial port connections or a combination of both. The network infrastructure to support each method of communication is illustrated in Figure 6 and Figure 7 respectively.

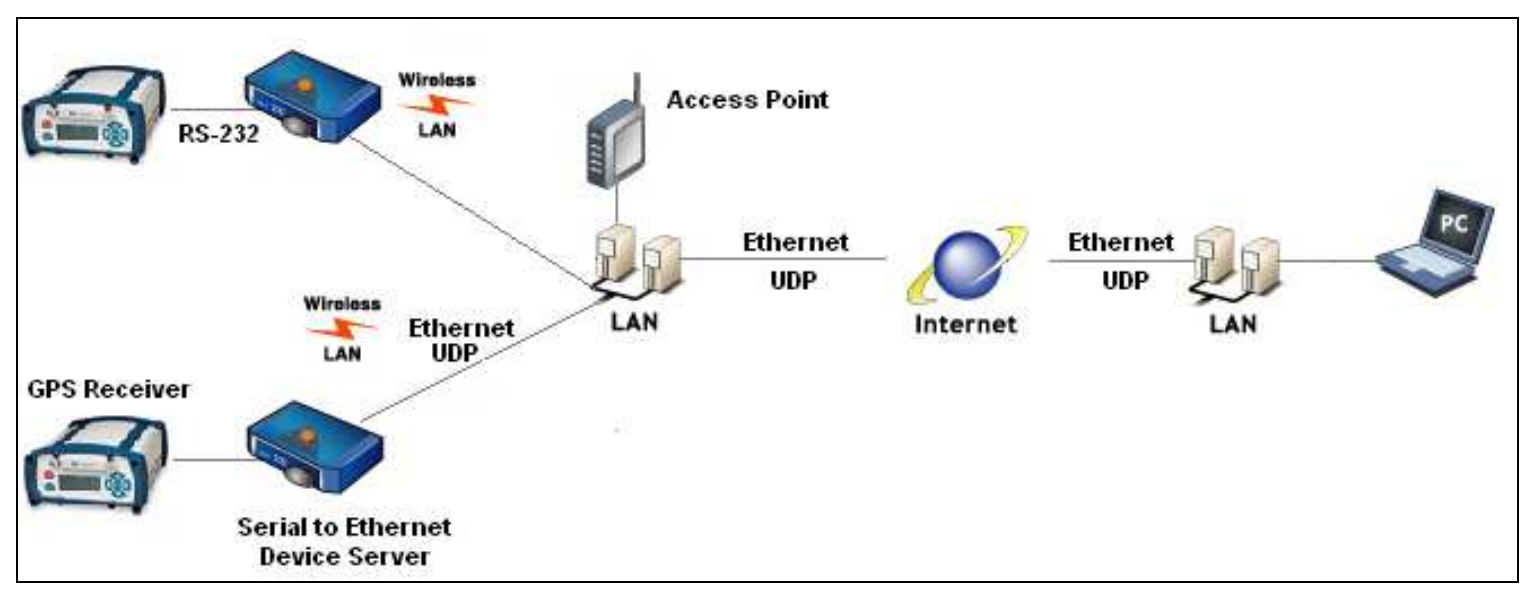

Figure 6. Ethernet based design (based on [11]).

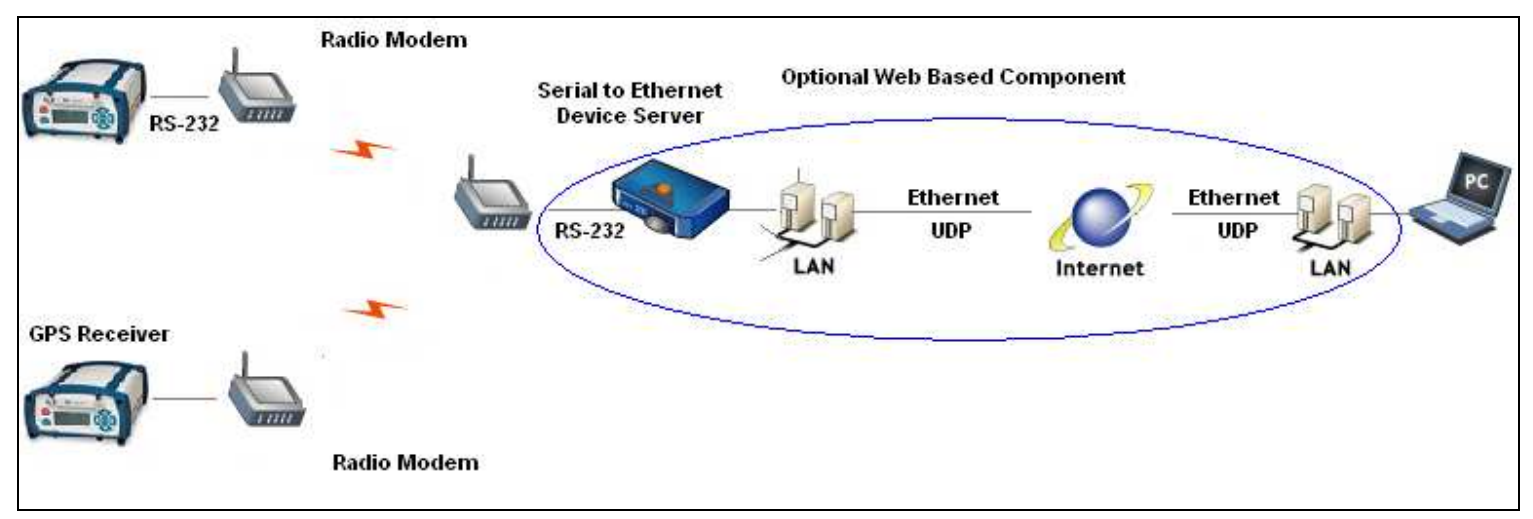

Figure 7. Radio Modem based Design.

In the first method, a serial-to-Ethernet device server (e.g., Sollae Systems EZL-400S) is used. Although the maximum data rate is limited here by the serial communication between the receiver and serial-to-Ethernet device server, new model GPS receivers (e.g., Novatel DL-V3, Trimble NetR7) offer direct Ethernet connection. Thus, this first method offers potentially higher data rates than the next to be discussed. Either LAN or WLAN can be used to communicate between the serial-to-Ethernet device server and the destination PC which will process the data. Local conditions and available broadband infrastructure will dictate which method is the most suitable to connect the GPS receiver to a wired network. 
The second method's design is similar to the first. An optional web based component is illustrated in Figure 7, which would allow for remote access of the data. A serial-to-Ethernet device server would be required to take advantage of this option so that the 'receive' radio modem could send its data online. Otherwise the data is directly streamed into the destination PC via serial port.

\subsubsection{PPMS Modifications}

In order to receive data packets in real-time from the GPS receiver, 'listening' threads are employed in the software to decode each receiver's binary data packets. In the case of an Ethernet based system, advantage is taken of the 'socket' class offered in the .NET development environment [12]. Various options for sockets exist as illustrated in Figure 8. "Asynchronous" sockets listen for incoming data on a separate thread without blocking operations on the main thread. "Synchronous" sockets are used for applications that only require one thread during execution since they block operations on other threads until their operations have completed. For this application, an asynchronous socket is preferred so that all incoming data is received.

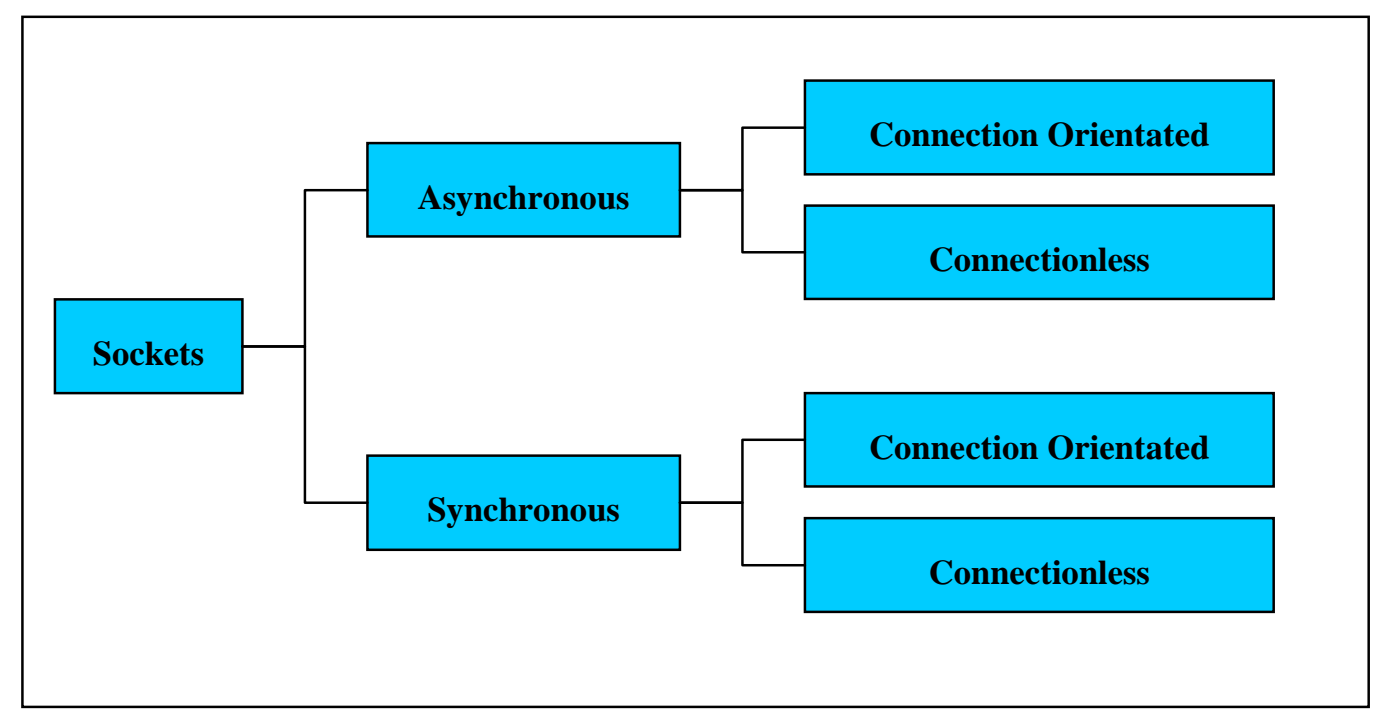

Figure 8. Types of Sockets.

Sockets can be further classified as "connection orientated" or "connectionless" depending upon the data transfer protocol. Connection orientated sockets can use Transmission Control Protocol (TCP) for transferring data. TCP requires a connection to be established with a remote endpoint and then uses that connection to send and receive data packets. An advantage of using TCP is that it ensures that data packets are sent to an endpoint and that they assembled in the correct order when they arrive [13]. A disadvantage of this approach is that there may be delays in ensuring that the correct data packets are being sent. These delays may not be tolerated when high data rates are used and latency must be minimized.

Connectionless sockets can use User Datagram Protocol (UDP) for transferring data. UDP offers the advantages of simplicity, quick data transfer and the ability to broadcast messages to multiple addresses at the same time. The disadvantage of this approach is that UDP datagrams are not 
guaranteed to arrive, nor are they guaranteed to arrive in the same sequence in which they were sent. Consequently, applications which use this protocol must be prepared to handle missing and out of sequence datagrams [14].

The concepts of TCP and UDP are illustrated in Figure 9 and Figure 10 respectively. It can be seen that prior to establishing a connection, all data sent by the GPS receiver are ignored in TCP mode. Once the connection is established, there is constant communication between the host GPS receiver and client PC.

In UDP mode, two parameters are used by the serial-to-Ethernet device server to determine when to send the GPS receiver's data: (1) a watermark value representing the maximum number of bytes that will be stored in its buffer before sending the data and (2) a timeout value that represents the length of time to wait after the first packet is received before sending the data. It does not matter whether or not there is a client listening. Once either one of these conditions is satisfied, the data stored in the serialto-Ethernet device server's buffer are sent.

For purposes of this application, UDP is the preferred method of communication due to: a) its ability to transfer data quickly and b) its simplicity, which makes it less susceptible to breakdowns in communication between client and host devices. The user must specify the IP address of the destination PC that will receive the data in the serial-to-Ethernet device server. The IP address of the serial-to-Ethernet device server must be specified in PPMS as illustrated in Figure 11.

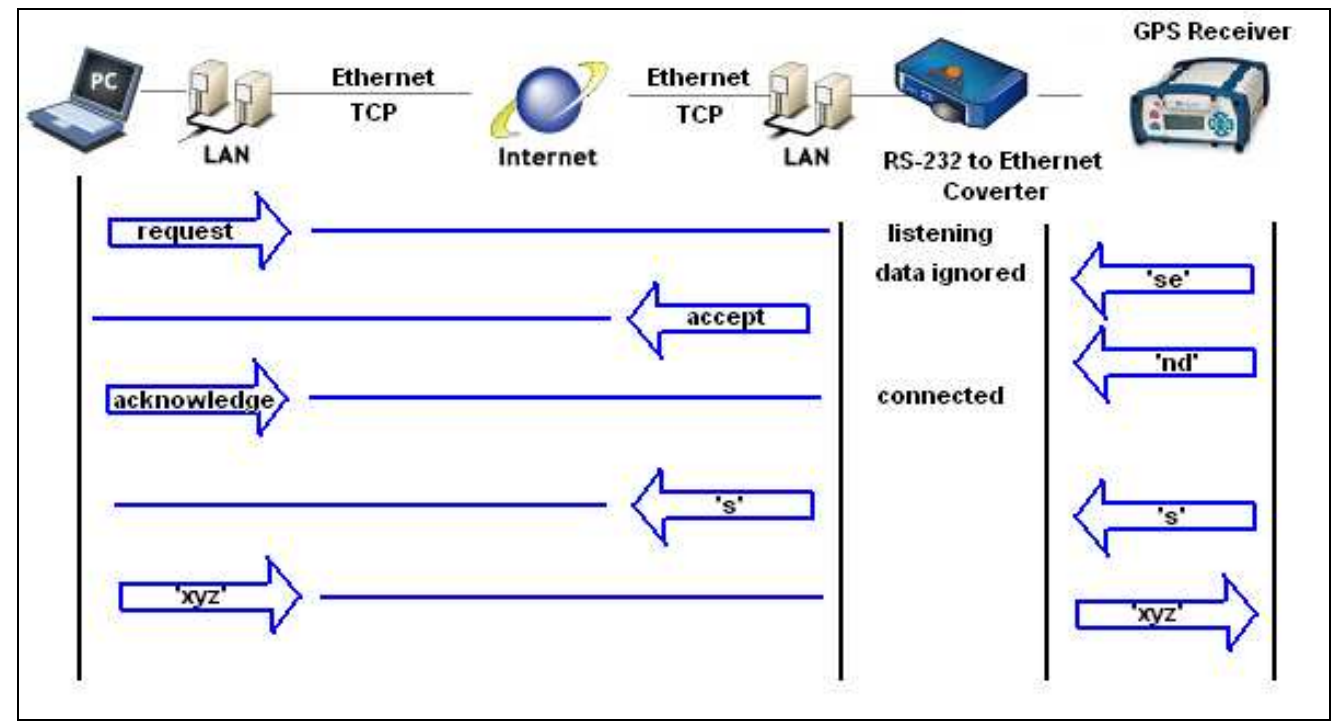

Figure 9. TCP Data Transfer Sequence (based upon [15]). 


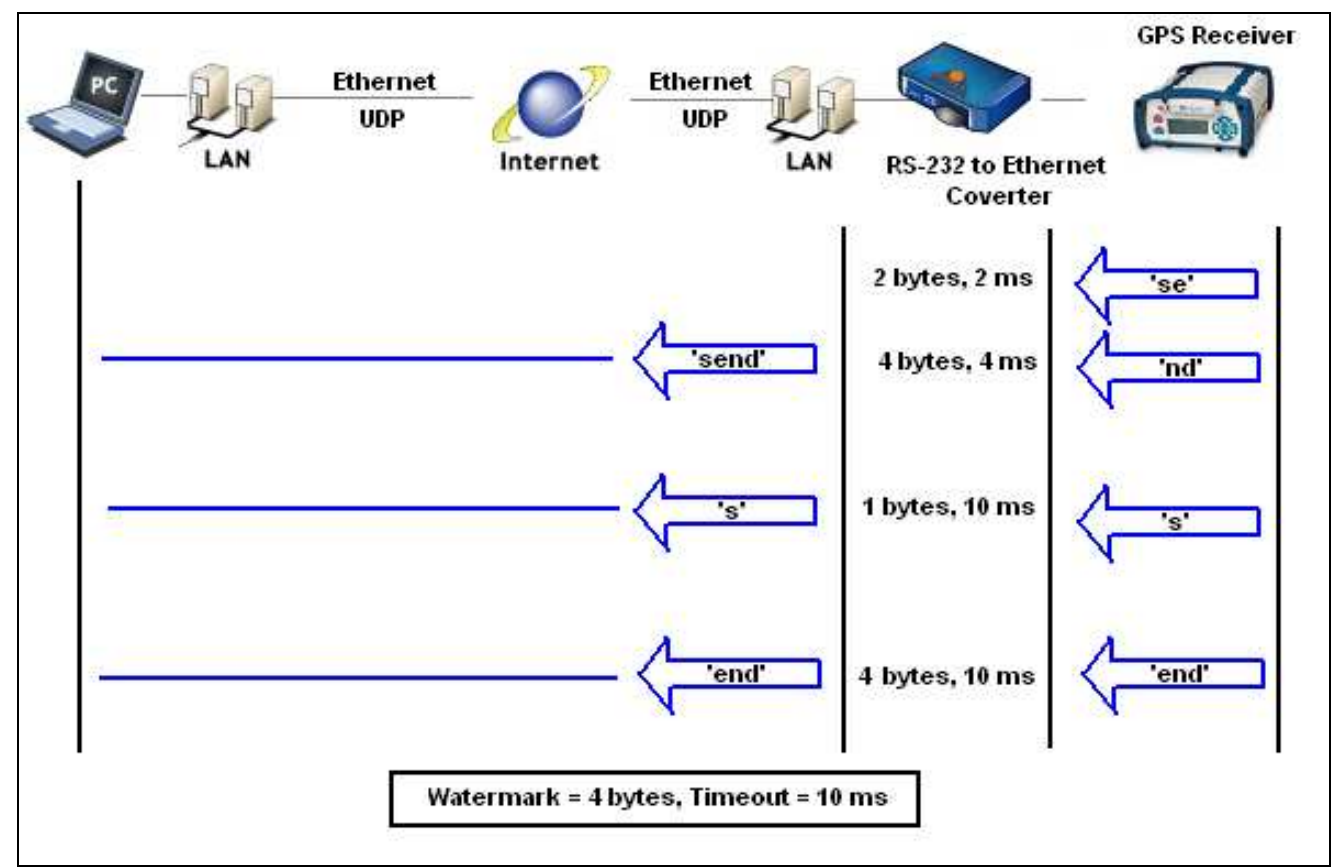

Figure 10. UDP Data Transfer Sequence (based upon [15]).

An asynchronous approach is also used for receiving data via serial port. A separate listening thread is invoked so that other operations are not blocked on the processing PC. Serial port settings are specified in PPMS software as illustrated in Figure 11.

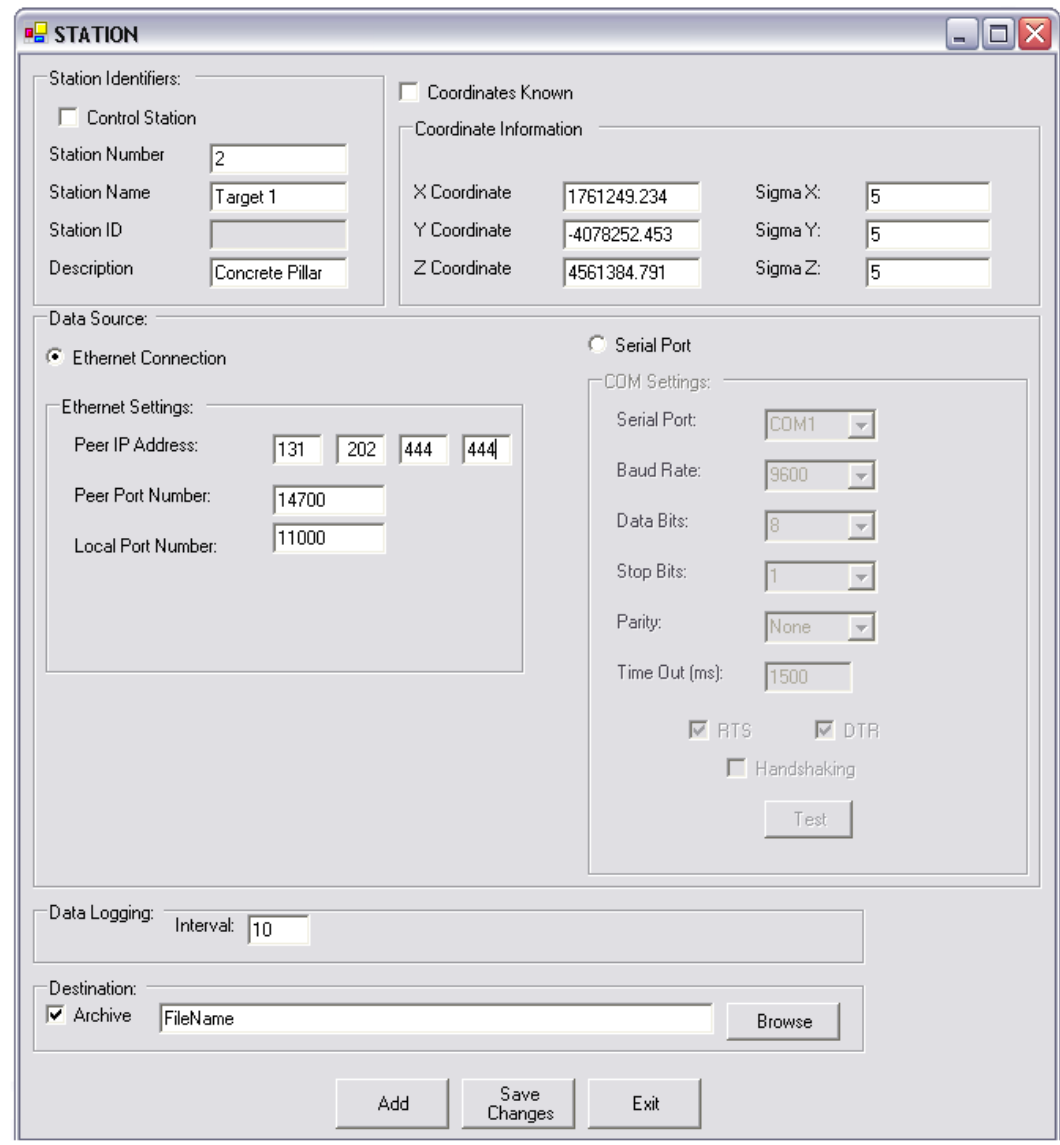

Figure 11. Adding a New GPS Station with Ethernet or Serial Port Connection. 


\subsubsection{Real-time Implementation}

To demonstrate the capabilities of the system, a real-time displacement detection test was performed. Two Novatel DL-4 GPS receivers with GPS-600 Pinwheel antennas were used to monitor the stability of a target point located on a translation stage. The target point was moved horizontally 10 mm. An IntegriNautics (now Novariant) IN200 pseudolite [16] was also used in this test to aid in quicker displacement detection [4]. The experiment configuration is illustrated in Figure 12.

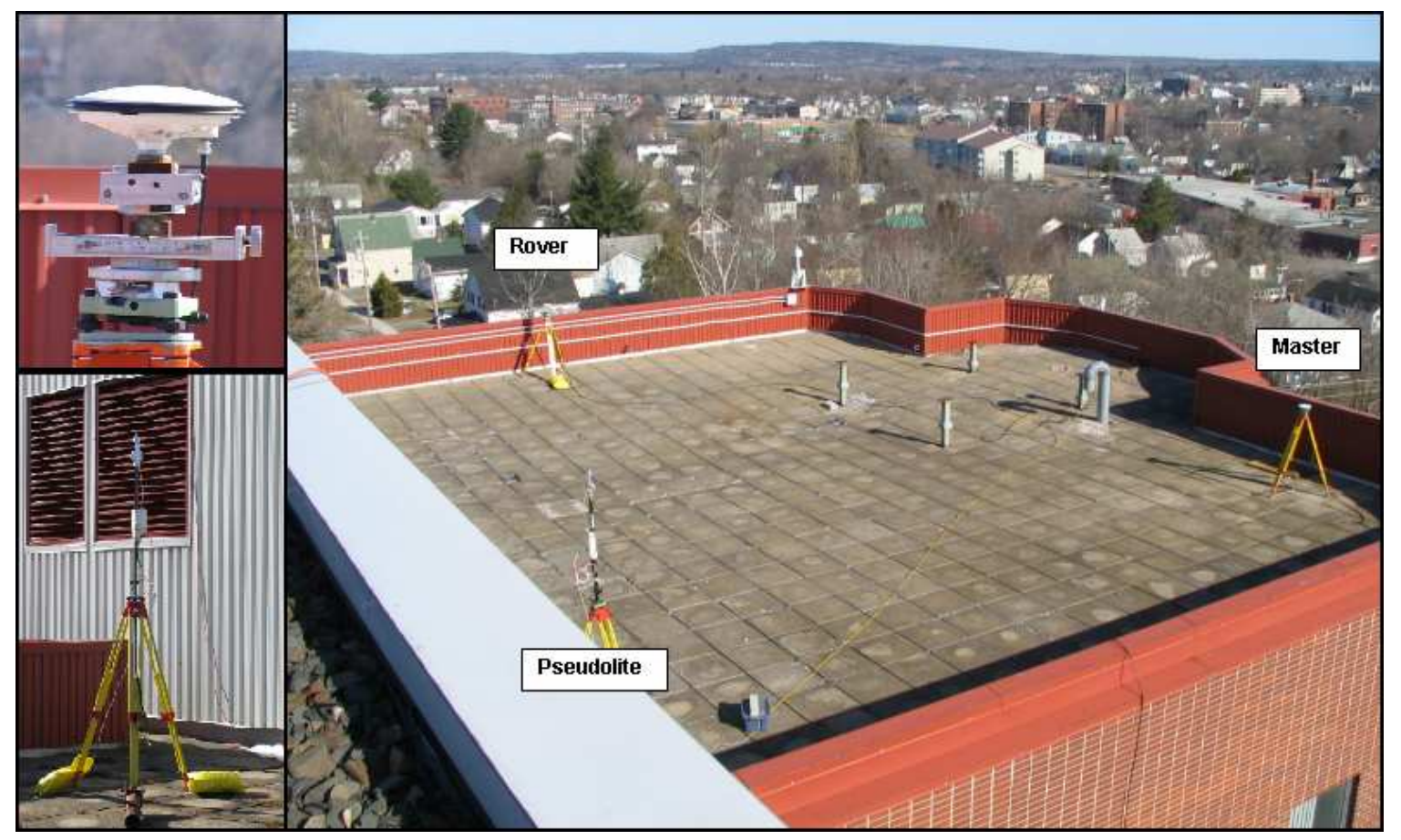

Figure 12. Displacement Detection Experiment Configuration (Top Left: Rover Antenna on Translation Stage, Bottom Left: IN200 Pseudolite, Right: Observed Baseline).

Two Sollae Systems serial-to-Ethernet device servers (model EZL-400S) were used to provide Ethernet connections to the receivers. A notebook PC with Intel Celeron $2.20 \mathrm{GHz}$ processor was used to run the software. The network infrastructure and system configuration are illustrated in Figure 13.

Initial tests of the monitoring system showed frequent gaps in the GPS data from each receiver and incomplete data packets being received. These problems were attributed to the watermark and timeout settings in the EZL-400S. If the watermark or timeout values were too small, the data would be sent to the remote $\mathrm{PC}$ as an incomplete data packet. The solution to this problem was to determine the maximum packet size that would be sent by the DL-4 receiver. The watermark value was then set to a value larger than the maximum packet size so that it would never trigger the data being sent. 


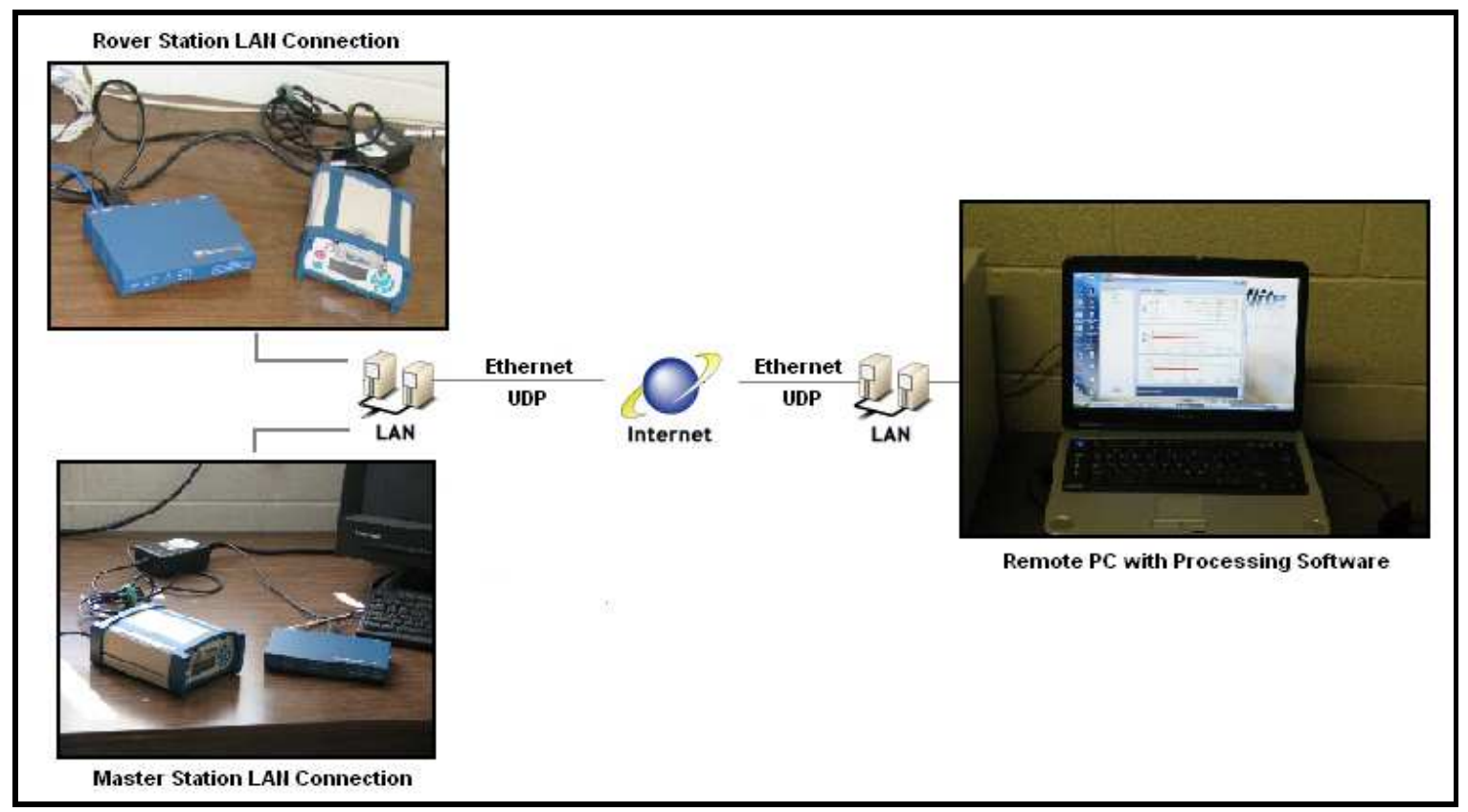

Figure 13. Network Infrastructure and System Configuration for Data Transfer and Remote Processing.

Based upon the baud rate of the DL-4 serial connection (57600 bps), the number of bits per packet (10: 1 start bit, 8 data bits and 1 stop bit) and the maximum data packet size (624 bytes) it was determined that approximately 5760 bytes / second could be sent or 5.76 bytes / millisecond. Accordingly, a timeout value of at least $110 \mathrm{~ms}$ was required. Since the timeout value of the EZL-400S has a precision of $\pm 10 \mathrm{~ms}$ [17], a timeout value of $120 \mathrm{~ms}$ was used.

Because data is transmitted in UDP mode, the EZL-400S sends data regardless of whether or not the remote PC is listening. It is necessary to allow sufficient time for the PC to receive messages from each receiver. This is especially important in this case since the DL-4 receiver clocks are synchronized at microsecond level with GPS time (and therefore with each other). If the same timeout value is used for each receiver, it is possible that while the PC is busy receiving packets from one receiver it may miss packets sent by another receiver in UDP mode. It is therefore recommended to stagger the timeout values for each receiver.

Figure 14 illustrates the output of PPMS software on the remote PC. The horizontal scale on the position plots represents GPS second of week. Prior to moving the translation stage, the coordinates of the target point are Easting: $-14.075 \mathrm{~m}$, Northing: $0.403 \mathrm{~m}$ and Up: $0.015 \mathrm{~m}$. At $\sim 303325 \mathrm{~s}$ the $10 \mathrm{~mm}$ displacement is introduced. Approximately 1 hour later $(\sim 307000 \mathrm{~s})$ the displacement is reflected in the Easting and Northing solution plots. After 4 hours $(\sim 318000 \mathrm{~s})$ the coordinates of the target point are Easting: $-14.068 \mathrm{~m}$, Northing: $0.393 \mathrm{~m}$ and Up: $0.018 \mathrm{~m}$ indicating a total horizontal displacement of $12 \mathrm{~mm}$. This information can be used to signal an alarm if conditions are deemed to be unsafe. 


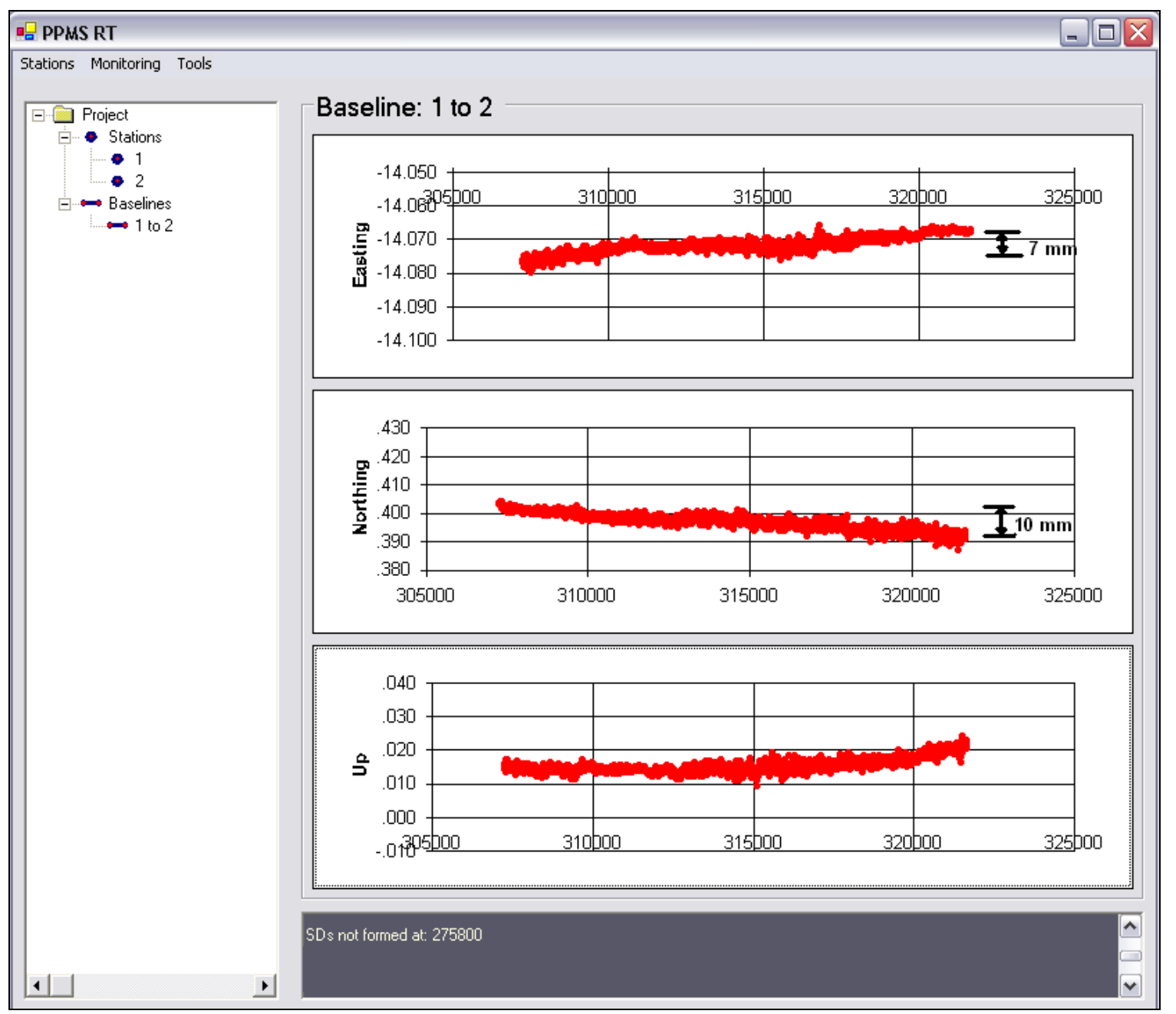

Figure 14. PPMS Graphical Display of Target Position.

\section{Conclusions}

A fully automated, continuous, real-time monitoring system has been developed that employs GPS sensors. The system is capable of providing sub-centimeter precisions without having to solve for the integer ambiguity, making it suitable for many deformation monitoring applications. Four major obstacles have had to be addressed to develop such a system. First, the effects of residual tropospheric delay biases have had to be mitigated from the height component of the solutions. This was addressed through the use of the TD observable over a high sample rate. Second, it was necessary to improve the continuity of the updates. Pseudolite technology was incorporated into the software to provide more frequent solutions. Third, a method of predicting the stability of reference points was required. It was shown how the results of deterministic modeling (and, in particular, the finite element method) could be used to achieve this end. Last, a fully-automated system was needed for providing continuous GPS position updates and this has been developed. Two communication options are currently available between GPS receivers at target points and a central processing computer: Ethernet or serial port. Both approaches allow the data to be processed locally or remotely based upon project needs.

The increasing number of catastrophes in recent years has lead to a demand for new sensors, sensor integration techniques and data processing strategies for improved deformation monitoring systems. 
Further research is required to integrate this technology with other sensors to create more reliable and more adaptable monitoring systems.

\section{Acknowledgements}

This research could not have been completed without the support of the Public Safety and Emergency Preparedness Canada Research Fellowship in honour of Stuart Nesbitt White, the Natural Sciences and Engineering Research Council of Canada (NSERC) and Atlantic Canada Opportunities Agency (ACOA). Special thanks to Sollae Systems Co., Ltd. for providing equipment necessary to conduct this research.

\section{References}

1. Bond, J.; Chrzanowski, A.; Wilkins, R. Using GPS for Augmenting Deformation Monitoring Systems in Open Pit Mines- Problems and Solutions. Geomatica 2005, 59(1), 73-82.

2. Chen, Y.Q.; Zhang, G.; Ding, X.; Li, Z. Monitoring Earth Surface Deformations with InSAR Technology: Principle and Some critical Issues. Journal of Geospatial Engineering 2000, 2(1), 321.

3. Kim, D.; Langley R.B; Bond, J.; Chrzanowski, A. Local deformation monitoring using GPS in an open pit mine: Initial study. GPS Solutions 2003 7(3): 176-185. DOI 10.1007/s10291-003-0075-1

4. Beutler, G.; Bauersima, I.; Gurtner, W.; Rothacher, M.; Schildknecht, T.; Gieger, A. Atmospheric refraction and other important biases in GPS carrier phase observations. Atmospheric Effects on Geodetic Space Measurements, Monograph 12, School of Surveying, University of New South Wales, pp. 15-43, 1988.

5. Langley, R.B. Propagation of the GPS Signals. Chapter 3 of GPS for Geodesy, Proceedings of the International School of GPS for Geodesy, Delft, the Netherlands, March 26 - April 1, 1995.

6. Bond, J.; Chrzanowski, A.; Kim, D. Bringing GPS into Harsh Environments for Deformation Monitoring. GPS Solutions 2007 (in press). Available at:

http://www.springerlink.com/content/109380/?sortorder=asc \&Content+Status=Accepted

7. Bond, J.; Chrzanowski, A.; Kim, D. Augmenting GPS with Pseudolites for Deformation Monitoring in Harsh Environments. Proceedings of the Institute of Navigation National Technical Meeting (ION NTM) 2007, 22-24 January, San Diego, CA, USA. Available at: http://www.ion.org/ and on CDROM.

8. Bond, J.; Szostak-Chrzanowski, A.; Chrzanowski, A. Design of Geodetic Monitoring Schemes Using Deterministic Modelling: An Open Pit Mine Example." Proceedings of the $3^{\text {rd }}$ International Symposium on Geo-information for Disaster Management, Toronto, Ontario, Canada, 22-24 May, Canadian Inst. of Geomatics, 2007. Available on CD-ROM.

9. Remondi, B.W. Using the Global Positioning System (GPS) Phase Observable for Relative Geodesy: Modeling, Processing, and Results. Doctoral thesis, Center for Space Research, University of Texas at Austin, pp. 1-324, 1984.

10. Brown, R.G.; Hwang, P.Y.C. Introduction to Random Signals and Applied Kalman Filtering, $3^{\text {rd }}$ ed., John Wiley \& Sons, Inc., New York, USA, pp. 1-484, 1997. 
11. Sollae Systems Co., Ltd. Products. EZL-400S. Sollae Systems Co., Ltd. website, 2007, http://www.eztcp.com/en/Products/ezl-400s.php, accessed on 18 April.

12. Microsoft Developer Network (MSDN). System.net.sockets namespace. Microsoft website, 2007, http://msdn2.microsoft.com/en-us/library/system.net.sockets(vs.71).aspx , accessed on 18 April.

13. Microsoft Developer Network (MSDN). Using TCP Services. Microsoft website, 2007, http://msdn2.microsoft.com/en-us/library/k8azesy5.aspx, accessed on 19 April.

14. Microsoft Developer Network (MSDN). Using UDP Services. Microsoft website, 2007, http://msdn2.microsoft.com/en-us/library/tst0kwb1.aspx, accessed on 19 April.

15. Sollae Systems Co., Ltd. Products EZL-400S: User's Manual. Sollae Systems Co., Ltd. website, 2007, http://www.eztcp.com/Support/ezl400sen.pdf, accessed on 18 April.

16. Novariant. Mining Products. Terralite XPS. Novariant website, 2007, http://www.novariant.com/mining/products/index.cfm, accessed on 21 April.

17. Lee, M. Email communication with Technical Support Representative, Sollae Systems Co., Ltd., 2007, 30 March.

(C) 2007 by MDPI (http://www.mdpi.org). Reproduction is permitted for noncommercial purposes. 